\title{
Immune response and endocytosis pathways are associated with the resilience against Alzheimer's disease
}

\author{
Niccolò Tesi $\mathbb{B}^{1,2,3}$, Sven J. van der Lee ${ }^{1,2}$, Marc Hulsman ${ }^{1,2,3}$, Iris E. Jansen ${ }^{1,4}$, Najada Stringa (1) ${ }^{5,6}$, \\ Natasja M. van Schoor ${ }^{5,6}$, Philip Scheltens ${ }^{1}$, Wiesje M. van der Flier ${ }^{1,5}$, Martijn Huisman ${ }^{5,6}$, Marcel J. T. Reinders (1) ${ }^{3}$ and \\ Henne Holstege $\mathbb{1}^{1,2,3}$
}

\begin{abstract}
Developing Alzheimer's disease (AD) is influenced by multiple genetic variants that are involved in five major ADpathways. Per individual, these pathways may differentially contribute to the modification of the AD-risk. The pathways involved in the resilience against AD have thus far been poorly addressed. Here, we investigated to what extent each molecular mechanism associates with (i) the increased risk of $A D$ and (ii) the resilience against $A D$ until extreme old age, by comparing pathway-specific polygenic risk scores (pathway-PRS). We used 29 genetic variants associated with $A D$ to develop pathway-PRS for five major pathways involved in AD. We developed an integrative framework that allows multiple genes to associate with a variant, and multiple pathways to associate with a gene. We studied pathway-PRS in the Amsterdam Dementia Cohort of well-phenotyped AD patients $(N=1895)$, Dutch population controls from the Longitudinal Aging Study Amsterdam $(N=1654)$ and our unique 100-plus Study cohort of cognitively healthy centenarians who avoided AD $(N=293)$. Last, we estimated the contribution of each pathway to the genetic risk of $A D$ in the general population. All pathway-PRS significantly associated with increased AD-risk and (in the opposite direction) with resilience against $A D$ (except for angiogenesis, $p<0.05$ ). The pathway that contributed most to the overall modulation of AD-risk was $\beta$-amyloid metabolism (29.6\%), which was driven mainly by APOEvariants. After excluding APOE variants, all pathway-PRS associated with increased AD-risk (except for angiogenesis, $p<$ $0.05)$, while specifically immune response $(p=0.003)$ and endocytosis $(p=0.0003)$ associated with resilience against AD. Indeed, the variants in these latter two pathways became the main contributors to the overall modulation of genetic risk of $A D(45.5 \%$ and $19.2 \%$, respectively). The genetic variants associated with the resilience against AD indicate which pathways are involved with maintained cognitive functioning until extreme ages. Our work suggests that a favorable immune response and a maintained endocytosis pathway might be involved in general neuro-protection, which highlight the need to investigate these pathways, next to $\beta$-amyloid metabolism.
\end{abstract}

Correspondence: Henne Holstege (h.holstege@amsterdamumc.nl)

${ }^{1}$ Alzheimer Center Amsterdam, Department of Neurology, Amsterdam

Neuroscience, Vrije Universiteit Amsterdam, Amsterdam UMC, Amsterdam, The Netherlands

2Department of Clinical Genetics, Amsterdam UMC, Amsterdam, The Netherlands

Full list of author information is available at the end of the article

\section{Introduction}

Owing to changes in lifestyle and advances in healthcare, life expectancy has greatly increased during the last century $^{1}$. A consequence of an increased fraction of aged individuals in the population is the increased prevalence of age-related diseases. A major contribution to poor health and disability at old age is cognitive decline due to Alzheimer's disease $(\mathrm{AD})^{2}$. The incidence of $\mathrm{AD}$ increases

\section{(c) The Author(s) 2020}

(c) Open Access This article is licensed under a Creative Commons Attribution 4.0 International License, which permits use, sharing, adaptation, distribution and reproduction c. in any medium or format, as long as you give appropriate credit to the original author(s) and the source, provide a link to the Creative Commons license, and indicate if changes were made. The images or other third party material in this article are included in the article's Creative Commons license, unless indicated otherwise in a credit line to the material. If material is not included in the article's Creative Commons license and your intended use is not permitted by statutory regulation or exceeds the permitted use, you will need to obtain permission directly from the copyright holder. To view a copy of this license, visit http://creativecommons.org/licenses/by/4.0/. 
exponentially with age and reaches $\sim 40 \%$ per year at 100 years, making it one of the most prevalent diseases in the elderly $^{3}$. Yet, a small proportion of the population $(<0.1 \%)$ avoids the disease, reaching at least 100 years while maintaining a high level of cognitive health ${ }^{4}$.

Both the development and the resilience against $\mathrm{AD}$ are determined by a combination of beneficial and harmful environmental and genetic factors that is unique for each individual $^{1,5,6}$. Thus far, large collaborative genome-wide association studies (GWAS) have discovered common genetic variants associated with a small modification of the risk of $\mathrm{AD}^{7-20}$. Of these, the alleles that encompass the $A P O E$ gene explain the largest proportion of the risk to develop or the chance to escape AD. We previously showed that those who avoided cognitive decline until extreme ages (cognitively healthy centenarians) were relatively depleted with genetic variants associated with an increased risk of $\mathrm{AD}^{21}$. However, the degree of depletion of these variants in the genomes of cognitively healthy centenarians relative to the middle-aged healthy individuals was not constant, which might point towards a differential impact of associated biological pathways on either avoiding or developing AD. This led us to hypothesize that an individuals' chance to develop $\mathrm{AD}$ or to being resilient against $\mathrm{AD}$ may be determined by pathway-specific risk.

Previous studies indicated that five specific biological pathways associate strongly with $\mathrm{AD}$ risk: immune response, $\beta$-amyloid metabolism, cholesterol/lipid dysfunction, endocytosis, and angiogenesis ${ }^{22-27}$. However, the extent to which different pathways contribute to the polygenic risk of $\mathrm{AD}$ is unknown. The degree to which a pathway contributes to the individual risk can be studied with pathway-specific polygenic risk scores (PRS ${ }^{28,29}$. In a typical PRS, the effect-sizes of all genetic variants that significantly associate with a trait are combined ${ }^{30}$. In a pathway-specific PRS, additional information is necessary: (i) the association of genetic variants to genes, and (ii) the association of genes to pathways. Previous studies of pathway-PRS in AD approached these challenges using the closest gene for variant mapping. For this, a 1:1 relationship between variants and genes is assumed, however, as AD-associated variants are mostly intronic or intergenic, the closest gene is not necessarily the gene affected by the variant. Additionally, different databases often have different functional annotations of genes, and this uncertainty was previously not taken into account when constructing pathway- $\mathrm{PRS}^{28,29}$.

An accurate mapping of the genetic risk of $\mathrm{AD}$ conferred by specific molecular pathways may lead to a greater comprehension of individual $\mathrm{AD}$ subtypes and might represent a first important step for the development of targeted intervention strategies and personalized medicine $^{31}$. Here, we propose a novel integrative framework to construct pathway-PRS for the five major pathways suggested to be involved in $\mathrm{AD}$. We then tested whether specific pathways differentially contributed to the risk of $\mathrm{AD}$, as well as to the chance of avoiding $\mathrm{AD}$ until extreme old ages. Finally, we estimated the contribution of each pathway to the polygenic risk of AD in the general (healthy middle-aged) population.

\section{Methods \\ Populations}

Population subjects are denoted by $P$ : they consist of a representative Dutch sample of 1779 individuals aged 55-85 years from the Longitudinal Aging Study Amsterdam (LASA) ${ }^{32,33}$. Patients diagnosed with AD are denoted by $A$. The patients are either clinically diagnosed probable AD patients from the Amsterdam Dementia Cohort $(N=$ 1630 ) or pathologically confirmed $A D$ patients from the Netherlands Brain Bank $(N=436)^{34-36}$. Escapers of AD are denoted by $C$ : these are 302 cognitively healthy centenarians from the 100-plus Study cohort. This study includes individuals who can provide official evidence for being aged 100 years or older and self-report to be cognitively healthy, which is confirmed by a proxy ${ }^{4}$. All participants and/or their legal representatives provided written informed consent for participation in clinical and genetic studies. The Medical Ethics Committee of the Amsterdam UMC (METC) approved all studies.

\section{Genotyping and imputation}

We selected 29 common genetic variants (minor allele frequency $>1 \%$ ) for which a genome-wide significant association with clinically identified $\mathrm{AD}$ cases was found (Supplementary Table S1) ${ }^{7-18,37-40}$. We genotyped all individuals using Illumina Global Screening Array (GSAsharedCUSTOM_20018389_A2) and applied established quality control measures ${ }^{4}$. Briefly, we used highquality genotyping in all individuals (individual call rate $>98 \%$, variant call rate $>98 \%$ ) and Hardy-Weinberg equilibrium-departure was considered significant at $p<$ $1 \times 10^{-6}$. Genotypes were prepared for imputation using provided scripts (HRC-1000G-check-bim.pl) ${ }^{42}$. This script compares variant ID, strand and allele frequencies to the haplotype reference panel (HRC v1.1, April 2016) ${ }^{35}$. Finally, all autosomal variants were submitted to the Michigan imputation server (Error! Hyperlink reference not valid.). The server uses SHAPEIT2 (v2.r790) to phase data and imputation to the reference panel (v1.1) was performed with Minimac3. Variant-genotypes of total of 1779 population subjects, 302 centenarians and $2052 \mathrm{AD}$ cases passed quality control. Prior to analysis, we excluded individuals of non-European ancestry $\left(N_{C}=2, N_{P}=63\right.$, and $N_{A}=94$ based on 1000Genomes clustering) ${ }^{43}$ and individuals with a family relation $\left(N_{C}=7, N_{P}=62\right.$, and $N_{A}=63$, identity-by-descent $\left.>0.3\right)$, leaving 1654 
population subjects, 293 cognitively healthy centenarians, and $1895 \mathrm{AD}$ cases for the analyses.

\section{Polygenic risk score}

To calculate the personal PRSs, or the genetic risk of $\mathrm{AD}$ that affects a single individual, the effect-sizes of all genetic variants that significantly associate with $\mathrm{AD}$ are combined. Formally, a PRS is defined as the sum of traitassociated alleles carried by an individual across a defined set of genetic loci, weighted by effect-sizes estimated from a GWAS ${ }^{30}$. We constructed a PRS using 29 variants that were previously associated with $\mathrm{AD}$. As weights for the PRS, we used the variant effect-sizes (log of odds ratio) as published in large GWAS of AD (Supplementary Table S1). Given a subject $s$, the PRS is defined as:

$$
\mathrm{PRS}^{s}=\sum_{K}^{K}\left(\operatorname{dos}_{k}^{s} * \beta_{k}\right),
$$

where $K$ is the full set of variants, $\operatorname{dos}_{k}^{s}$ is the allele dosage from the (imputed) genotype of variant $k$ in subject $s$ and $\beta_{k}$ is the effect-size as determined in the largest published AD case-control GWAS (Supplementary Table S1).

\section{Mapping variants to pathways}

We studied the five pathways implicated in AD: immune response, $\beta$-amyloid metabolism, cholesterol/lipid dysfunction, endocytosis, and angiogenesis ${ }^{22-25,44,45}$. For these pathways we developed the variant-pathway mapping $M_{p}^{k}$, which represents the degree of involvement of a given variant in the pre-selected pathways. To generate this value, we $(i)$ associated genetic variants to genes (variant-gene mapping), (ii) associated genes to pathways (gene-pathway mapping), and (iii) combined these mappings in the variant-pathway mapping.

Variant-gene mapping: the association of a variant with a specific gene is not straight-forward as the closest gene is not necessarily the gene affected by the variant. The two most recent and largest GWAS of AD addressed the relationship between genetic variants and associated genes applying two independent methods ${ }^{19,20}$. Briefly, one study used (i) gene-based annotation, (ii) expressionquantitative trait loci (eQTL) analyses, (iii) gene cluster/ pathway analyses, and (iv) differential gene expression analysis between $\mathrm{AD}$ cases and healthy controls ${ }^{19}$. The other study integrated (i) positional mapping, (ii) eQTL gene-mapping, and (iii) chromatin interaction as implemented in the tool Functional Mapping and Annotation of Genome-Wide Association Studies (FUMA) ${ }^{20,46}$. The list of genes most likely affected by each variant was obtained from both studies and used to derive a weighted mapping for each genetic variant $k$ to one or more genes $g$, $m_{g}^{k}$, denoted as the variant-gene-mapping weight. This weight was calculated by counting the number of times a variant $k$ was associated with gene $g$ across the two studies and dividing this by the total number of genes associated with the variant (Supplementary Table S2). For variants in/near CR1, PILRA, PLCG2, ABCA7 and APOE, we assumed the culprit gene as known, and we assigned a 1:1 relationship between the variant and the gene (Supplementary Table S2).

Gene-pathway mapping: each gene from the variantgene mapping was classified into the pre-defined set of pathways integrating four sources of information:

1. Gene-sets from the unsupervised pathway enrichment analysis within MAGMA statistical framework from Kunkle et al. ${ }^{19}$, in which the authors identified nine significant pathways (coupled with the genes involved in each pathway), which we mapped to 3 of the 5 pathways of interest (Supplementary Table S3);

2. Associated genes from Gene-ontology (GO, from AmiGO 2 version 2.5.12, released on 2018-04) terms resembling the five pathways of interest within the biological processes tree (including all child-terms) (Supplementary Table S4) ${ }^{47,48}$;

3. Gene-sets derived from an unsupervised functional clustering analysis within DAVID (v6.8, released on $2016-10)^{49,50}$ : the gene-set from the variant-gene mapping was used to obtain 12 functional clusters, which were then mapped to the 5 pre-selected pathways using a set of keywords (Supplementary Tables S5 and S6);

4. Gene-pathway associations from a recent review concerning the genetic landscape of $\mathrm{AD}$ (Supplementary Table S7) ${ }^{22}$;

By counting the number of times each gene was associated to each pathway according to these sources, and dividing by the total number of associations per gene, we obtained a weighted mapping of each gene $g$ to one or more pathways $p, w_{p}^{g}$, denoted as the gene-pathwaymapping weight (Supplementary Tables S8 and S9). In case the gene-pathway mapping could not be calculated (i.e., there was no mapping to any of the pathways of consideration), we excluded the gene from further analyses (Supplementary Tables S8 and S9).

To associate variants with pathways, we combined the variant-gene mapping and the gene-pathway mapping. Given a variant $k$, mapping to a set of genes $G$, and a pathway $p$, we define the weight of the variant to the pathway $\left(M_{p}^{k}\right)$ as:

$$
M_{p}^{k}=\sum_{g}^{G}\left(m_{g}^{k} * w_{p}^{g}\right)
$$

where $m_{g}^{k}$ is the variant-gene-mapping weight of variant $k$ to gene $g$, and $w_{p}^{g}$ is the gene-pathway-mapping weight of 
gene $g$ to pathway $p$. In this way, for each variant, we calculated a score indicative of the involvement of the variant in each of the five pathways (variant-pathway mapping, Supplementary Table S10). For some variants no variant-pathway-mapping was possible. We marked these variants as unmapped (Supplementary Table S10).

\section{Pathway-specific PRS}

For the pathway-specific PRS (pPRS), we extended the definition of the PRS by adding as multiplicative factor the variant-pathway-mapping weight of each variant. Given a sample $s$ and a pathway $p$, we defined the pPRS as:

$$
\operatorname{pPRS}_{p}^{s}=\sum_{k}^{K}\left(\operatorname{dos}_{k}^{s} * \beta_{k} * M_{k}^{p}\right),
$$

where $M_{k}^{p}$ is the variant-pathway mapping of variant $k$ to pathway $p$.

\section{Association of PRSs in the three cohorts}

We calculated the PRS and pathway-PRS (pPRS) for the population subjects, the $\mathrm{AD}$ cases and the cognitively healthy centenarians ( $P, A$, and $C$, respectively). Prior to analyses, the PRSs of all three populations were combined together and were scaled (mean $=0, \mathrm{SD}=1$ ). We then investigated the influence of $A P O E$, gender and age on the risk scores: we calculated the PRSs and pPRSs with and without the two $A P O E$ variants and we correlated the resulting (p)PRSs with sex, age (age at inclusion for controls, age at onset for cases), and population substructure components. To inspect the differential contributions of the risk scores to $\mathrm{AD}$ development or resilience against $\mathrm{AD}$, we calculated $(i)$ the association of the risk scores (PRS and pPRS) with AD status by comparing AD cases and population subjects $(A v s . P)$, and (ii) the association of the risk scores with resilience against AD by comparing cognitively healthy centenarians and population subjects (C vs. $P$ comparison). For the associations, we used logistic regression models with the PRS and pPRS as predictors, adjusting for population substructure (principal components 1-5). Resulting effect-sizes (log of odds ratio) can be interpreted as the odds ratio difference per one standard deviation (SD) increase in the PRS, with a corresponding estimated 95\% confidence intervals (95\% $\mathrm{CI})$. Association analyses of the (p)PRS in the three population were also stratified by sex. Last, we verified the classification performances of the single variants, as well as the (p)PRS by calculating the area under the ROC curve for classification of $\mathrm{AD}$ and resilience against $\mathrm{AD}$.

\section{Comparison of effect-size between resilience against $A D$ and increased AD-risk}

To further investigate the relationship between the effect of each pathway on $\mathrm{AD}$ and on resilience against
$\mathrm{AD}$, we calculated the change in effect-size. This corresponds to the ratio between the effect-size of the association with resilience against $\mathrm{AD}(\log$ of odds ratios of $C$ vs. $P$ comparison) and the effect-size of the association with AD (log of odds ratios of $A$ vs. $P$ comparison). We calculated the change in effect-size for the pPRS, including and excluding $A P O E$ variants. We estimated 95\% confidence intervals for the effect-size ratios by sampling, and we tested for significant difference between the change in effect-size, including and excluding $A P O E$ variants (respectively, for each of pPRS) using $t$-test. A value for the change in effect-size of 1 indicates a similar effect on increased risk of $\mathrm{AD}$ and resilience against $\mathrm{AD}$. Although a value for the change in effect-size unknown a priori, since all variants considered are selected to be associated with $\mathrm{AD}$, a value $<1$ is expected (i.e., a larger effect on $\mathrm{AD}$ than on resilience against $\mathrm{AD}$ ).

\section{Contribution of each pathway to polygenic risk of $A D$}

We estimated the contribution of each pathway to the genetic risk of $\mathrm{AD}$ in the general population: this equals to the variance explained by each of the pre-selected pathways to the genetic risk of AD. Mathematically, this is the ratio between the variance of each pathway-PRS and the variance of the combined PRS as calculated in the individuals in the general population. As such, it is a function of the variant-pathway mapping, the effect-size (log of odds ratio) of the variants, and the variant frequencies. Given a variant $k$ and the relative variant-pathway mapping $M_{k}^{p}$, we define the percentage of the risk explained by each pathway $p$ as:

$$
P^{p}=\frac{\sum_{k}^{K}\left(M_{k}^{p} * \beta_{k}^{2} * \operatorname{MAF}_{k} *\left(1-\operatorname{MAF}_{k}\right)\right)}{\sum_{k}^{K}\left(\beta_{k}^{2} * \operatorname{MAF}_{k} *\left(1-\mathrm{MAF}_{k}\right)\right)},
$$

where $\beta_{k}$ is the variant effect-size from literature, and $\mathrm{MAF}_{k} *\left(1-\mathrm{MAF}_{k}\right)$ is the variance of a Bernoulli random variable that occurs with probability $\mathrm{MAF}_{k}$, i.e., the minor allele frequency of each variant $k$ in our cohort of population subjects. Here, $M_{k}^{p}$ is interpreted as the probability that variant $k$ belongs to pathway $p$. Importantly, for each variant, $\sum_{P}^{P} M_{k}^{p}=1$, so that each variant contributes equally, yet differentially at the level of each pathway. This means that the variance of a variant is only counted once, even if the variant contributes to multiple pathways. When calculating the contributions of each pathway, we also considered variants with missing variant-pathway mapping. For these variants, the variant-pathway mapping was set to 1 for an unmapped pathway. Altogether, the pathway-PRS variances sum to the total PRS variance.

\section{Implementation}

We performed quality control of genotype data, as well as population stratification analysis and relatedness 
analysis with PLINK (v2.0). All subsequent analyses were performed with $\mathrm{R}$ (v3.5.2), Bash and Python (v2.7.14) scripts. We provide a R script to construct pPRS and PRS using our variant-pathway annotation and user's genotypes. In addition, all the scripts we used to perform the analyses can be found at https://github.com/TesiNicco/ pathway-PRS.

\section{Results}

After quality control of the genetic data, we included 1654 population subjects (with mean age at inclusion $62.7 \pm 6.4,53.2 \%$ females), $1895 \mathrm{AD}$ cases (with mean age at onset $69.2 \pm 9.9,56.4 \%$ females), and 293 cognitively healthy centenarians (with mean age at inclusion 101.4 \pm $1.3,72.6 \%$ females) ( $P, A$, and $C$, respectively).

\section{PRSs associate with $A D$ and escape from $A D$}

To each subject, we assigned a PRS representative of all $29 \mathrm{AD}$-associated variants, including and excluding $A P O E$ variants. We found that the PRS, when including $A P O E$ variants, significantly associated with an increased risk of $\mathrm{AD}$ and, in the opposite direction, with increased chance of resilience against $\mathrm{AD}(A$ vs. $P$ : $\mathrm{OR}=2.61,95 \% \mathrm{CI}=$ [2.40-2.83], $p=8.4 \times 10^{-113}$ and $C$ vs. $P: \mathrm{OR}=0.54,95 \%$ $\mathrm{CI}=[0.45-0.65], p=1.1 \times 10^{-10}$ ) (Fig. $1 \mathrm{a}$ and Supplementary Table S11). When excluding APOE variants, the PRS was still significantly associated with an increased risk of $\mathrm{AD}$ and, in the opposite direction, with increased risk of resilience against $\mathrm{AD}(A$ vs. $P$ : $\mathrm{OR}=1.30,95 \%$ $\mathrm{CI}=[1.22-1.40], p=3.1 \times 10^{-14}$ and $C$ vs. $P: \mathrm{OR}=0.78$, 95\% CI $=[0.69-0.89], p=2.4 \times 10^{-4}$ ) (Fig. 1b and Supplementary Table S11).

\section{Pathway-specific PRS associate with AD and escape from AD}

We annotated the $29 \mathrm{AD}$-associated genetic variants to 5 selected pathways (Fig. 2). According to our variantgene mapping, the $29 \mathrm{AD}$-associated variants mapped to 110 genes (Supplementary Table S8). The number of genes associated with each variant ranged from 1 (e.g., for variants in/near CR1, PILRA, SORL1, ABCA7, APOE, or $P L C G 2$ ), to 30 (a variant in the gene-dense region within the $H L A$ region) (Fig. 2 and Supplementary Table S8). We were able to calculate the gene-pathway-mapping weight for 69 genes (Supplementary Table S9). The remaining 41 genes were not mapped to the 5 pathways. In total, we calculated the variant-pathway mapping for 23 loci to at least one of the pre-selected biological pathways (Fig. 2 and Supplementary Table S10).

We then calculated the pPRS for each pathway in population subjects, $\mathrm{AD}$ cases and cognitively healthy centenarians, including and excluding $A P O E$ variants (Fig. $1 b, c)$. The number of variants that contributed to each pPRS was 19 for immune response, 11 for $\beta$-amyloid metabolism, 19 for endocytosis, 8 for cholesterol/lipid dysfunction, and 4 for angiogenesis pathways (Supplementary Tables S10 and S11). Overall, the pPRS (including and excluding the $A P O E$ variants) positively and significantly correlated with each other and with the overall PRS (Supplementary Fig. S1), and did not correlate with gender and age (Supplementary Fig. S1).

When including $A P O E$ variants, the pPRSs of all pathways (except for angiogenesis) significantly associated with increased risk of $\mathrm{AD}$, independently from gender $(A v s . P$, immune response: $\mathrm{OR}=2.15,95 \% \mathrm{CI}=[1.99-2.32], p=$ $2.0 \times 10^{-80} ; \beta$-amyloid metabolism: $\mathrm{OR}=2.52,95 \% \mathrm{CI}=$ [2.32-2.73], $p=7.8 \times 10^{-109}$; endocytosis: OR $=2.55,95 \%$ $\mathrm{CI}=[2.35-2.77], \quad p=1.7 \times 10^{-109} ;$ cholesterol/lipid dysfunction: $\mathrm{OR}=2.55,95 \% \mathrm{CI}=[2.35-2.76], \quad p=2.1 \times$ $10^{-110}$; angiogenesis: $\mathrm{OR}=1.05,95 \% \mathrm{CI}=[0.98-1.12]$, $p=0.134$ ) (Fig. 1b, Supplementary Table S11, Supplementary Fig. S2, and Supplementary Table S12). The association of pPRSs with increased chance of being resilient against $\mathrm{AD}$ was in the opposite direction for all pathways, and the association was significant for all pathways except for angiogenesis ( $C$ vs. $P$, immune response: $\mathrm{OR}=0.64,95 \% \mathrm{CI}=[0.54-0.74], p=1.4 \times 10^{-8}$; $\beta$-amyloid metabolism: $\mathrm{OR}=0.59,95 \% \mathrm{CI}=[0.49-0.71]$, $p=2.7 \times 10^{-8} ; \quad$ endocytosis: $\quad \mathrm{OR}=0.55, \quad 95 \% \quad \mathrm{CI}=$ [0.46-0.66], $p=1.3 \times 10^{-10}$; cholesterol/lipid dysfunction: $\mathrm{OR}=0.58,95 \% \mathrm{CI}=[0.48-0.70], p=1.8 \times 10^{-8} ;$ angiogenesis: $\mathrm{OR}=0.90,95 \% \mathrm{CI}=[0.79-1.01], p=0.078$ ) (Fig. $1 \mathrm{~b}$ and Supplementary Table S11). Directions of effects were consistent in both males and females, but the significance of associations was reduced due to stratification (Supplementary Table S12 and Supplementary Fig. S2).

When excluding $A P O E$ variants, the pPRSs of all pathways (except for the angiogenesis) was still significantly associated with increased risk of AD without specific gender effects $(A$ vs. $P$, immune response: $\mathrm{OR}=1.19,95 \%$ $\mathrm{CI}=[1.11-1.27], p=5.5 \times 10^{-7} ; \beta$-amyloid metabolism: $\mathrm{OR}=1.19,95 \% \mathrm{CI}=[1.12-1.28], p=2.0 \times 10^{-7} ;$ endocytosis: $\mathrm{OR}=1.27,95 \% \mathrm{CI}=[1.19-1.36], p=2.8 \times 10^{-12}$; cholesterol/lipid dysfunction: $\mathrm{OR}=1.18, \quad 95 \% \quad \mathrm{CI}=$ [1.11-1.27], $p=7.5 \times 10^{-7}$; angiogenesis: $\mathrm{OR}=1.05,95 \%$ $\mathrm{CI}=[0.98-1.12], \quad p=0.134$ ) (Fig. 1c, Supplementary Table S11, Supplementary Fig. S2, and Supplementary Table S12). The association of pPRSs with increased chance of being resilient against AD was in the opposite direction for all pathways, yet the association was significant only for the immune response and the endocytosis pPRS ( $C$ vs. $P$, immune response: $\mathrm{OR}=0.82,95 \% \mathrm{CI}=$ [0.72-0.94], $p=0.003 ; \beta$-amyloid metabolism: $\mathrm{OR}=0.91$, $95 \% \mathrm{CI}=[0.80-1.03], p=0.131$; endocytosis: $\mathrm{OR}=0.79$, 95\% $\mathrm{CI}=[0.70-0.90], \quad p=2.8 \times 10^{-4} ;$ cholesterol/lipid dysfunction: $\mathrm{OR}=0.91,95 \% \mathrm{CI}=[0.80-1.03], p=0.145$; angiogenesis: $\mathrm{OR}=0.90,95 \% \mathrm{CI}=[0.79-1.01], p=0.078)$ (Fig. 1c and Supplementary Table S11). 


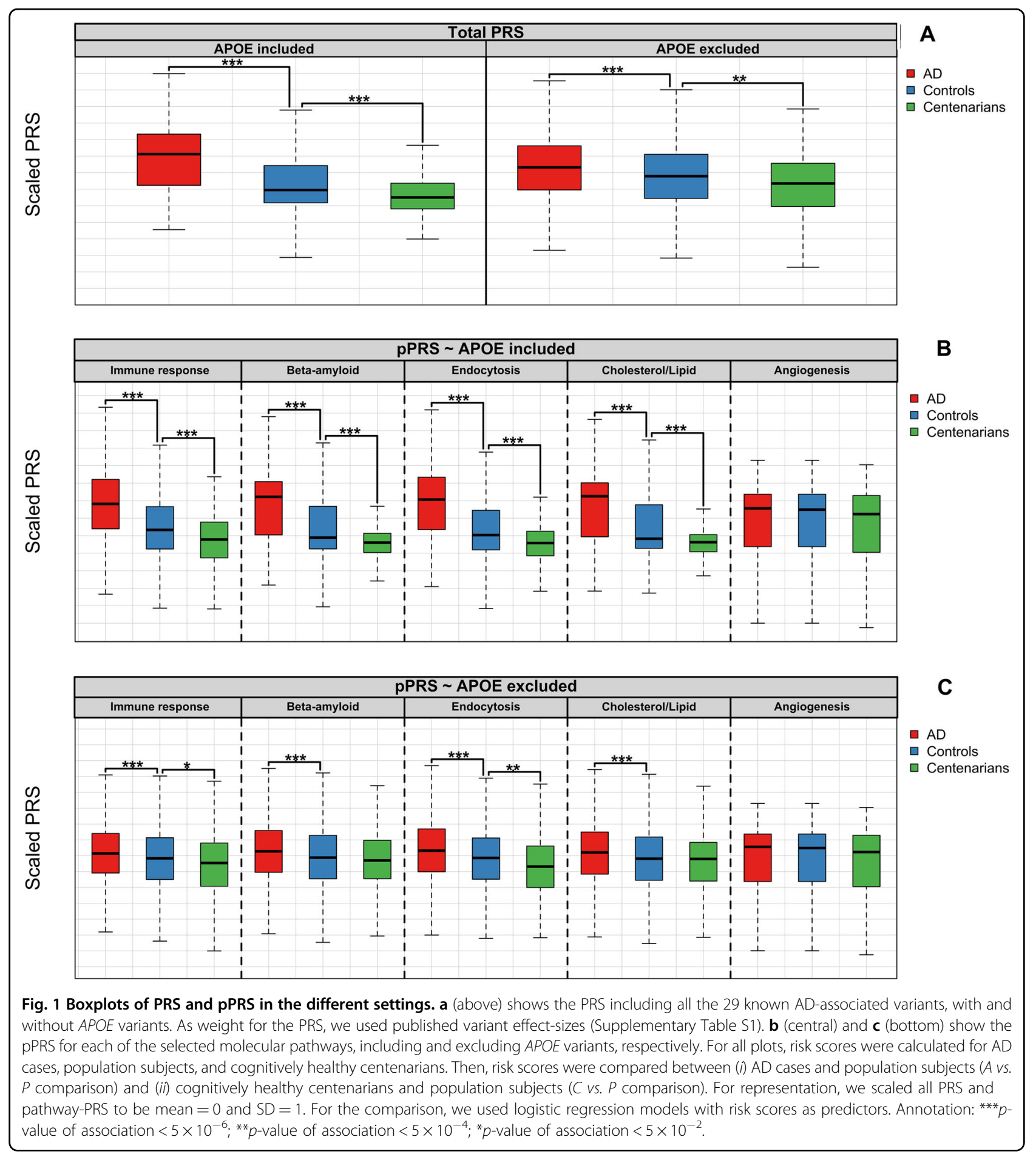

In the sex-stratified analysis, females reported consistent direction of effects and significant associations of immune response and endocytosis pathways, while in males the direction was consistent for immune response, endocytosis and angiogenesis pathways, and it was opposite for $\beta$-amyloid metabolism and cholesterol/lipid dysfunction (yet not significant) (Supplementary Fig. S2 and Supplementary Table S12).

We note that apart from $A P O E$ variants (for which we stratified the analyses for), there was no major driver in the pPRS, as well as the single-variant associations (Supplementary Figs. S3 and S4). 


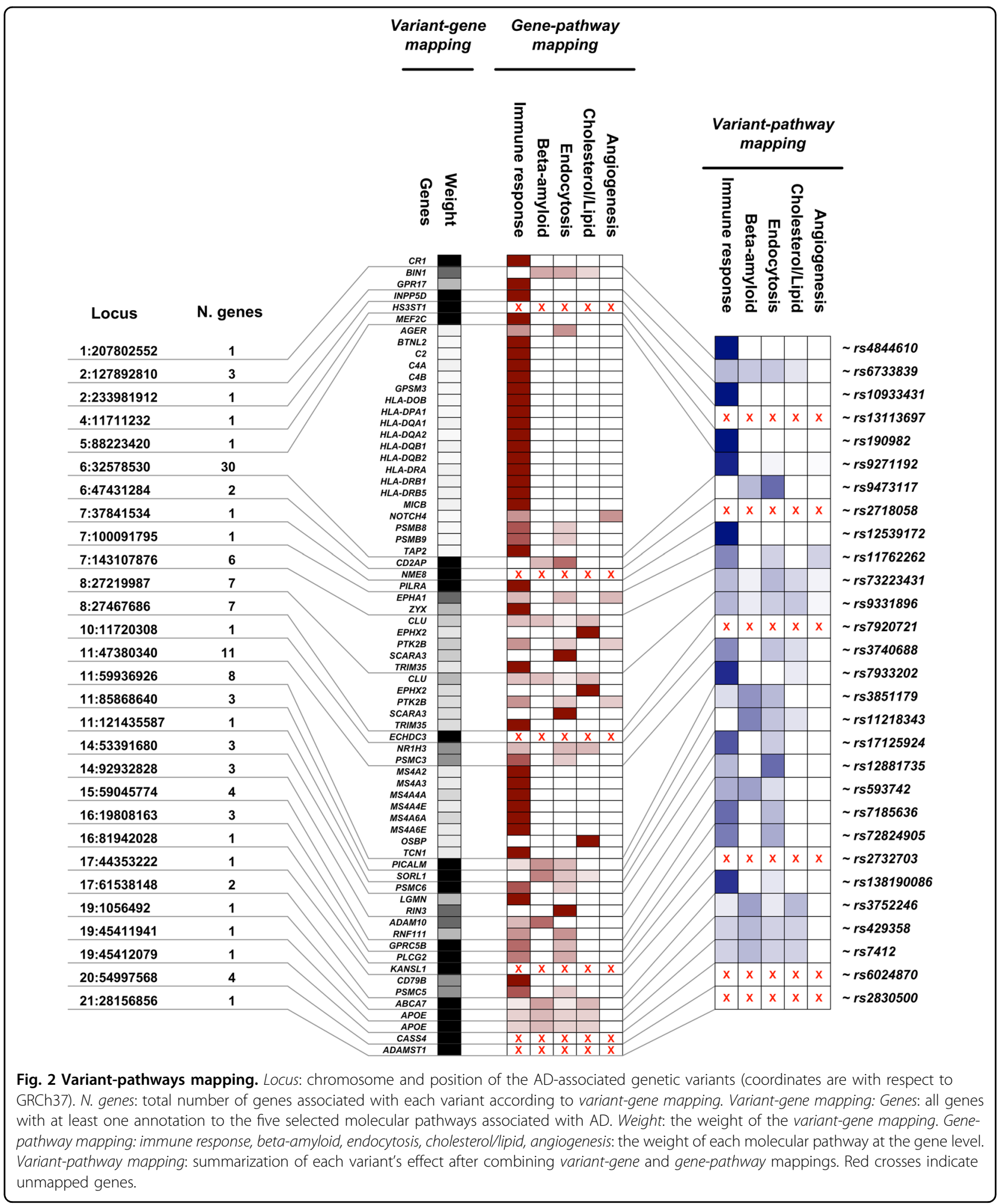

\section{Comparison of effect on $A D$ and escaping $A D$}

To further evaluate the association of the pPRSs with $\mathrm{AD}$ and with resilience against $\mathrm{AD}$, we compared, for each pPRS, the reciprocal effect-size associated with resilience against $\mathrm{AD}$ with the effect-size associated with increased risk of AD (change in effect-size, Fig. 3a). When including $A P O E$ variants, the change in effect-size was $<1$ for all pathways (except for the angiogenesis pathway) (Fig. 3b). 


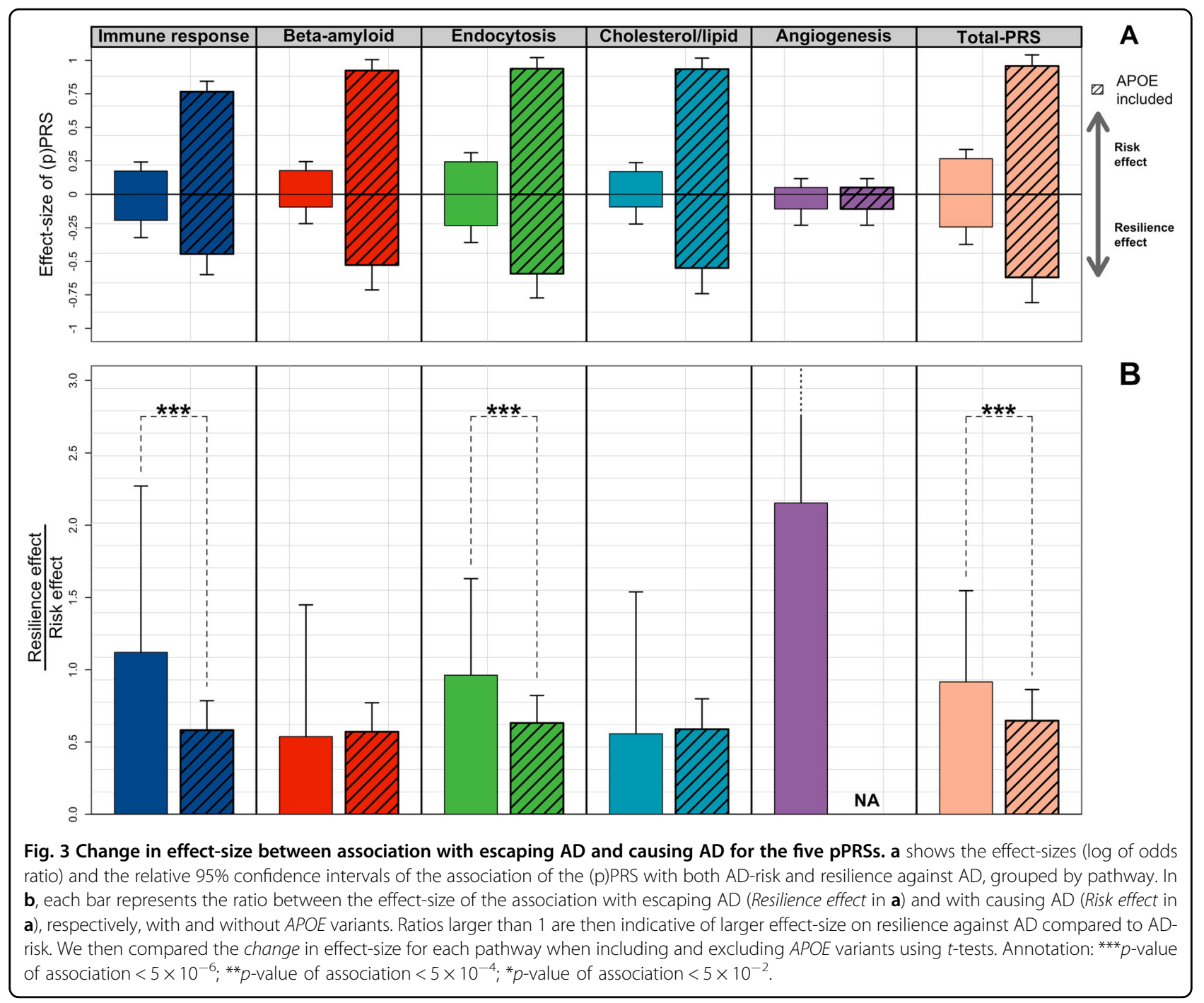

This is expected as the effect-size of $A P O E$ variants on causing $\mathrm{AD}$ is much larger than its effect on resilience against $\mathrm{AD}$ (Fig. 3a). When excluding $A P O E$ variants, the change in effect-size was still $<1$ for $\beta$-amyloid metabolism and cholesterol/lipid metabolism (respectively, 0.54 and 0.58 ), but it approximated 1 for endocytosis (0.96) and it was larger than 1 for the immune response and angiogenesis (respectively, 1.12 and 2.15) (Fig. 3b). Interestingly, we found that the relative effect-size for immune response and endocytosis excluding $A P O E$ variants was significantly higher than that including $A P O E$ variants $\left(p<2.1 \times 10^{-197}\right.$ and $p<$ $8.9 \times 10^{-180}$, respectively), suggesting a larger effect on resilience against $\mathrm{AD}$ compared to $\mathrm{AD}$-risk for these pathways, specifically when excluding $A P O E$ variants (Fig. $3 \mathrm{~b}$ ).

\section{Contributions of each pathway to the polygenic risk of $A D$}

Finally, we estimated the relative contribution of each pathway to the polygenic risk of $\mathrm{AD}$ in the general population. This is indicative of the degree of involvement of each pathway to the total polygenic risk of $\mathrm{AD}$, and as such it is based on our variant-pathway mapping. Including APOE variants, the contribution of the pathways to the total polygenic risk of $\mathrm{AD}$ was $29.6 \%$ for $\beta$-amyloid metabolism, $26.6 \%$ for immune response, $21.6 \%$ for endocytosis, $19.5 \%$ for cholesterol/lipid dysfunction, $0.3 \%$ for angiogenesis, and 2.3\% for the unmapped variants (Fig. 4a).

When we excluded $A P O E$ variants, the contribution of the pathways to the total polygenic risk of AD was $45.5 \%$ for immune response, $19.2 \%$ for endocytosis, $13.7 \%$ for $\beta$ amyloid metabolism, 8\% for cholesterol/lipid dysfunction, $1.4 \%$ for angiogenesis and $12.3 \%$ for the unmapped variants (Fig. 4b).

\section{Discussion}

In this work, we studied 29 common genetic variants known to associate with AD using PRSs and pathwayspecific PRSs. As expected, we found that a higher PRS for $A D$ was associated with a higher risk of AD. Previous 


\section{APOE included}

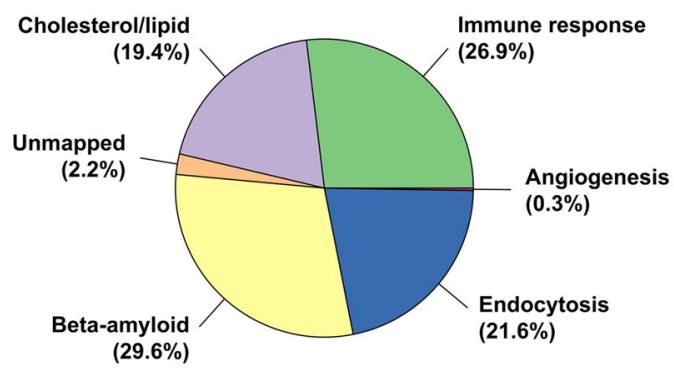

APOE excluded

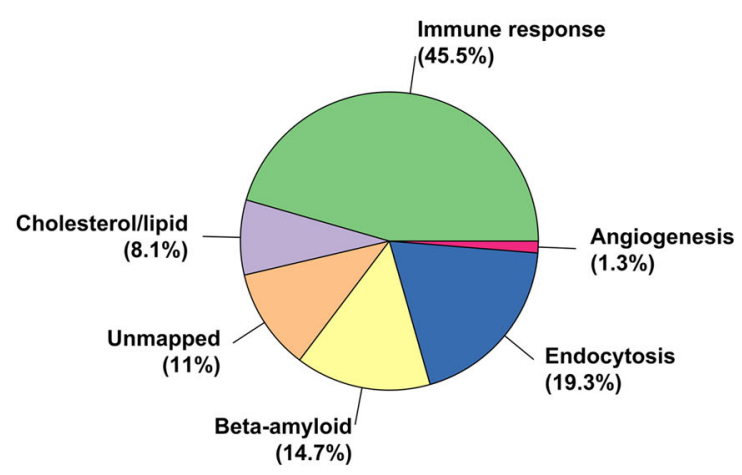

Fig. 4 Explained variance of each pathway-specific PRS to polygenic risk of AD. The pie charts represents the explained variance of each pathway-specific PRS to the polygenic risk of AD, including and excluding APOE variants. The contributions are calculated according to (i) our variantpathway mapping, (ii) the effect-size (log of odds ratio) of each variant from literature (Supplementary Table S1), and (iii) variant's frequency in our cohort of middle-aged healthy population subjects. We also considered variants with missing variant-pathway mapping (unmapped pathway).

studies showed that PRS of AD not only associated with increased risk of $\mathrm{AD}$, but also with neuropathological hallmarks of $\mathrm{AD}$, lifetime risk and the age at onset in both $A P O E \& 4$ carriers and non-carriers ${ }^{28,29,51-55}$. We now add that, using our unique cohort of cognitively healthy centenarians, the PRS for $\mathrm{AD}$ also associates with resilience against $\mathrm{AD}$ at extremely old ages. This adds further importance to the potentiality of using PRS and $A P O E$ genotype in a clinical setting ${ }^{51,52,54,56}$. In addition, our analyses suggest that the long-term preservation of cognitive health is associated with the selective survival of individuals with the lowest burden of risk-increasing variants or, vice versa, the highest burden of protective variants.

Using an innovative approach, we studied five pathways previously found to be involved in $\mathrm{AD}$, as well as the contribution of these pathways to the polygenic risk of AD. We showed that all pathways-PRS except angiogenesis associate with increased $\mathrm{AD}$ risk, both including and excluding APOE variants and independently from gender. When we studied the association of pathways-PRS with resilience against $\mathrm{AD}$ until extreme old ages, we found that, as expected, the enrichment of the protective $A P O E$ $\varepsilon 2$ allele and the depletion of the risk-increasing $A P O E \varepsilon 4$ allele represented a major factor in avoiding AD. However, when excluding the two $A P O E$ variants, only immune response and endocytosis significantly associated with an increased chance to be resilient against AD. Interestingly, both pathways had a larger or similar effect on resilience against $\mathrm{AD}$-resilience compared to developing $\mathrm{AD}$, suggesting that these pathways might be involved in general neuro-protective functions. Based on the variant effect-size, variant frequency and our variantpathway mapping, we found that the $\beta$-amyloid metabolism (29.6\%) followed by immune response (26.6\%) were the major contributors to general modification of $\mathrm{AD}$ - risk. After excluding $A P O E$ variants, according to our analysis, immune response (45.5\%) and endocytosis (19.2\%) contributed most to the modification of AD-risk.

Our approach to map variants to associated genes and to map genes to pathways resulted in a weighted annotation of variants to pathways that allowed for uncertainty in gene as well as pathway assignment, which was not done previously. We note that considering uncertainty in variant-gene as well as gene-pathway assignments is crucial because most genetic variants are in non-coding regions, which makes the closest gene not necessarily the culprit gene, and because different functional annotation sources often do not overlap. In our variant-pathway mapping, a larger number of annotations (both variantgenes and gene-pathways), generally causes a dilution of the "true" variant effect, reflecting increasing uncertainty in the annotation sources used. This depends on the specific regions, for example, the HLA region carries many genes with large linkage signals, however, all genes in this region are typically annotated with immune response. We point out that the power of the PRSs does not only reflect the effect-size of the variants, but also the number and frequency of the variants that contribute to the PRSs: due to this, a larger number of very common variants with relatively small effect-size can still have more power (yet small ORs) than a small number of relatively rare variants with high effect-size. The pathwayspecific PRS that we proposed in this manuscript can be re-used for the identification of subtypes of $\mathrm{AD}$ patients compromised in a specific $\mathrm{AD}$-associated pathway. This is of interest for clinical trials, in order to test responsiveness to compounds in specific subsets of patients. For example, monoclonal antibody targeting TREM2 receptors could work better in AD patients who have an impaired immune response pathway. Recently, several studies 
attempted to construct pathway-specific PRS to find heterogeneity in AD patients based on a genetic basis ${ }^{28,29}$. In line with our findings, Ahmad et al. ${ }^{29}$ found that genes capturing endocytosis pathway significantly associated with $\mathrm{AD}$ and with the conversion to $\mathrm{AD}$. Other studies used less variants ${ }^{28}$ or less stringent selection for variants, and did not observe a differential involvement of pathways in $\mathrm{AD}$ etiology ${ }^{57}$.

The amyloid cascade hypothesis has been dominating AD-related research in the last two decades. However, treatments targeting amyloid have, so far, not been able to slow or stop disease progression. This has led to an increased interest for the other pathways that are important in $\mathrm{AD}$ pathogenesis ${ }^{22}$. Part of the current view of the etiology of $\mathrm{AD}$ is that the dysregulation of the immune response is a major causal pathway, and that $\mathrm{AD}$ is not only a consequence of $\beta$-amyloid metabolism ${ }^{58,59}$. In addition, previous studies showed that healthy immune and metabolic systems are associated with longer and healthier lifespan ${ }^{1,60}$. Our results indicate that, excluding $A P O E$ variants, the effect of immune response and endocytosis on escaping $\mathrm{AD}$ is stronger or comparable to the effect on causing AD. This suggests that these pathways might be involved in the maintenance of general cognitive health, as the cognitively healthy centenarians represent the escape of all neurodegenerative diseases until extreme ages. We recently found evidence for this hypothesis in the protective low-frequency variant in PLCG2, which is involved in the regulation of the immune response $^{53}$. This variant is enriched in cognitively healthy centenarians, and protects against $\mathrm{AD}$, as well as frontotemporal dementia and dementia with Lewy bodies ${ }^{53}$. We included this variant in the total PRS, as well as in the pathway-PRS for the immune response (variant-pathway mapping was 60\%) and endocytosis (variant-pathway mapping was 40\%). Regarding endocytosis, this pathway is thought to play a role both in neurons, as part of the $\beta$-amyloid metabolism, but also in glia cells, as part of the immune response. Thus, a dysregulation in the interplay between these pathways might lead to an imbalance of immune signaling factors, favoring the engulfment of synapses and $\mathrm{AD}$-associated processes. This, in turn, may contribute to the accumulation of amyloid and tau pathologies ${ }^{61-64}$.

We assessed the effect of common and low-frequency variants on the development and the escape of $\mathrm{AD}$. Therefore, the contributions of rare, causative variants associated with increased AD risk, such as those in $A P P$, PSEN1, PSEN2, TREM2, and SORL1 were not considered. Despite the large odds ratios to develop $A D$ associated with carrying such variants, the frequency of these variants in the population is ultra-low, and therefore have a minor effect on the total AD risk in the population ${ }^{11,12}$.
However, future versions of the PRS will most likely include the effect of carrying disease-associated rare variants. This will affect individual PRS scores and the necessity to accordingly adapt the results generated with current PRSs. Compared to the sizes of recent GWAS of $A D$, we included relatively small sample sizes, particularly with respect to the cognitively healthy centenarians, a very rare phenotype in the population $(<0.1 \%)^{4}$. These sample sizes are, however, sufficient to study PRSs. The cohorts that we used in this study were not used in any GWAS of $\mathrm{AD}$, therefore we provide independent replication of $\mathrm{AD}$ PRS in a homogeneous group of (Dutch) individuals.

We note that, apart from $A P O E$ variants (for which we stratify the analyses for), none of the other variants have been associated with longevity or well cognitive functioning in the largest and most recent GWAS ${ }^{65,66}$. We acknowledge that our variant-pathway mapping reflects the current state of imperfect knowledge at the level of AD-GWAS findings, variant-gene and gene-mechanism relationships. Thus, as new variants, pathways or functional relationships will be identified, the contributions and the pathway-specific PRSs will need to be recalculated. Of note: the study in which the genome-wide significant association with $\mathrm{AD}$ of the variant in/near KANSL1 was originally identified, reported a larger effectsize compared to the effect-size used in our manuscript ( $\beta=0.31$ and $\beta=0.07$, respectively), possibly because the original analysis was stratified by $A P O E$. We cannot exclude that we underestimated the contribution of KANSL1 in the analyses. Moreover, since the KANSL1 variant did not map into one of the analyzed pathways, it was not included in any of the pathway-specific PRS calculations. A limitation, not exclusive to our work, is the highly debated role of $A P O E$ gene. We mapped the effect of $A P O E$ to four pathways and we are aware this assignment is relatively arbitrary. We add that $A P O E$ has wellstudied (cardio)vascular properties that are included in our cholesterol and lipid metabolism pathway. The combination of a large effect and unclear pathway assignment makes that pathway-PRS, including APOE challenging to use. Lastly, we point out that the variance contributions might change in different populations, as it depends on variant frequency and population heterogeneity.

Concluding, with the exclusion of $A P O E$ variants and based on our functional annotation of variants, the aggregate contribution of the immune response and endocytosis represents $>60 \%$ of the currently known polygenic risk of $\mathrm{AD}$. This indicates that an intervention in these systems may have large potential to prevent $A D$ and potentially other related diseases and highlights the critical need to study (neuro)immune response and endocytosis, next to $\beta$-amyloid metabolism. 


\section{Acknowledgements}

The following studies and consortia have contributed to this manuscript Amsterdam dementia cohort (ADC): Research of the Alzheimer center Amsterdam is part of the neurodegeneration research program of Amsterdam Neuroscience (www.amsterdamresearch.org). The Alzheimer Center Amsterdam is supported by Stichting Alzheimer Nederland and Stiching VUmc fonds. The clinical database structure was developed with funding from Stichting Dioraphte. Genotyping of the Dutch case-control samples was performed in the context of EADB (European Alzheimer DNA biobank) funded by the JPco-fuND FP-829-029 (ZonMW projectnumber 733051061). 100-plus study: we are grateful for the collaborative efforts of all participating centenarians and their family members and/or relatives. This work was supported by Stichting Alzheimer Nederland (WE09.2014-03), Stichting Diorapthe, horstingstuit foundation, Memorabel (ZonMW projectnumber 733050814) and Stichting VUmc Fonds. Genotyping of the 100-plus study was performed in the context of EADB (European Alzheimer DNA biobank) funded by the JPco-fuND FP-829-029 (ZonMW projectnumber 733051061). The clinical database structure was developed with funding from Stichting Dioraphte. Longitudinal Aging Study Amsterdam (LASA) is largely supported by a grant from the Netherlands Ministry of Health, Welfare and Sports, Directorate of Long-Term Care. The authors are grateful to all LASA participants, the fieldwork team and all researchers for their ongoing commitment to the study. This work was in part carried out on the Dutch national e-infrastructure with the support of SURF Cooperative.

\section{Author details}

'Alzheimer Center Amsterdam, Department of Neurology, Amsterdam Neuroscience, Vrije Universiteit Amsterdam, Amsterdam UMC, Amsterdam, The Netherlands. 'Department of Clinical Genetics, Amsterdam UMC, Amsterdam, The Netherlands. ${ }^{3}$ Delft Bioinformatics Lab, Delft University of Technology, Delft, The Netherlands. ${ }^{4}$ Department of Complex Trait Genetics, Center for Neurogenomics and Cognitive Research, Amsterdam Neuroscience, VU University, Amsterdam, The Netherlands. ${ }^{5}$ Department of Epidemiology and Biostatistics, Amsterdam UMC, Amsterdam, The Netherlands. ${ }^{6}$ Amsterdam Public Health Research Institute, Amsterdam, The Netherlands

\section{Conflict of interest}

The authors declare that they have no conflict of interest.

\section{Publisher's note}

Springer Nature remains neutral with regard to jurisdictional claims in published maps and institutional affiliations.

Supplementary Information accompanies this paper at (https://doi.org/ 10.1038/s41398-020-01018-7).

Received: 22 November 2019 Revised: 14 July 2020 Accepted: 15 July 2020 Published online: 29 September 2020

\section{References}

1. Partridge, L., Deelen, J. \& Slagboom, P. E. Facing up to the global challenges of ageing. Nature 561, 45-56 (2018).

2. Alzheimer's Association. 2012 Alzheimer's disease facts and figures. Alzheimers Dement. 8, 131-168 (2012).

3. Corrada, M. M., Brookmeyer, R., Paganini-Hill, A., Berlau, D. \& Kawas, C. H. Dementia incidence continues to increase with age in the oldest old: The 90 + study. Ann. Neurol. 67, 114-121 (2010).

4. Holstege, $\mathrm{H}$. et al. The 100-plus Study of cognitively healthy centenarians: rationale, design and cohort description. Eur. J. Epidemiol. https://doi.org/ 10.1007/s10654-018-0451-3 (2018)

5. Niccoli, T. \& Partridge, L. Ageing as a risk factor for disease. Curr. Biol. 22 R741-R752 (2012)

6. Brooks-Wilson, A. R. Genetics of healthy aging and longevity. Hum. Genet. 132, 1323-1338 (2013).

7. Lambert, J. C. et al. Meta-analysis of 74,046 individuals identifies 11 new susceptibility loci for Alzheimer's disease. Nat. Genet. 45, 1452-1458 (2013).
8. Harold, D. et al. Genome-wide association study identifies variants at CLU and PICALM associated with Alzheimer's disease. Nat. Genet. 41, 1088-1093 (2009).

9. Seshadri, S. et al. Genome-wide analysis of genetic loci associated with Alzheimer disease. JAMA 303, 1832-1840 (2010).

10. Desikan, R. S. et al. Polygenic overlap between C-reactive protein, plasma lipids, and Alzheimer disease. Circulation 131, $2061-2069$ (2015)

11. Sims, R. et al. Rare coding variants in PLCG2, ABI3, and TREM2 implicate microglial-mediated innate immunity in Alzheimer's disease. Nat. Genet. 49, 1373-1384 (2017)

12. Guerreiro, R. et al. TREM2 variants in Alzheimer's disease. N. Engl. J. Med. 368 117-127 (2013).

13. Jonsson, T. et al. Variant of TREM2 associated with the risk of Alzheimer's disease. N. Engl. J. Med. 368, 107-116 (2013).

14. Hollingworth, P. et al. Common variants at ABCA7, MS4A6AMS4A4E, EPHA1, CD33 and CD2AP are associated with Alzheimer's disease. Nat. Genet. 43, 429-435 (2011).

15. Naj, A. C. et al. Common variants at MS4A4/MS4A6E, CD2AP, CD33 and EPHA1 are associated with late-onset Alzheimer's disease. Nat. Genet. 43, 436-441 (2011).

16. Jun, G. et al. A novel Alzheimer disease locus located near the gene encoding tau protein. Mol. Psychiatry 21, 108-117 (2016).

17. Steinberg, S. et al. Loss-of-function variants in ABCA7 confer risk of Alzheimer's disease. Nat. Genet. 47, 445-447 (2015).

18. Marioni RE, et al. GWAS on family history of Alzheimer's disease. Trans/ Psychiatry. 8, 99 (2018).

19. Alzheimer Disease Genetics Consortium (ADGC). et al. Genetic meta-analysis of diagnosed Alzheimer's disease identifies new risk loci and implicates $A \beta$, tau, immunity and lipid processing. Nat. Genet. 51, 414-430 (2019).

20. Jansen, I. E. et al. Genome-wide meta-analysis identifies new loci and functional pathways influencing Alzheimer's disease risk. Nat. Genet. 51, 404-413 (2019).

21. Tesi, N. et al. Centenarian controls increase variant effect sizes by an average twofold in an extreme case-extreme control analysis of Alzheimer's disease. Eur. J. Hum. Genet. https://doi.org/10.1038/s41431-018-0273-5 (2018)

22. Van Cauwenberghe, C., Van Broeckhoven, C. \& Sleegers, K. The genetic landscape of Alzheimer disease: clinical implications and perspectives. Genet. Med. 18, 421-430 (2016)

23. Hardy, J. et al. Pathways to Alzheimer's disease. J. Intern. Med. 275, 296-303 (2014).

24. Naj, A. C. \& Schellenberg, G. D., for the Alzheimer's Disease Genetics Consortium (ADGC). Genomic variants, genes, and pathways of Alzheimer's disease: an overview. Am. J. Med. Genet. B Neuropsychiatr. Genet. 174, 5-26 (2017).

25. Verheijen, J. \& Sleegers, K. Understanding Alzheimer disease at the interface between genetics and transcriptomics. Trends Genet. 34, 434-447 (2018).

26. Bennett, R. E. et al. Tau induces blood vessel abnormalities and angiogenesisrelated gene expression in P301L transgenic mice and human Alzheimer's disease. Proc. Natl Acad. Sci. 115, E1289-E1298 (2018).

27. Di Marco, L. Y. et al. Vascular dysfunction in the pathogenesis of Alzheimer's disease-A review of endothelium-mediated mechanisms and ensuing vicious circles. Neurobiol. Dis. 82, 593-606 (2015).

28. Darst, B. F. et al. Pathway-specific polygenic risk scores as predictors of amyloid- $\beta$ deposition and cognitive function in a sample at increased risk for Alzheimer's disease. J. Alzheimers Dis. 55, 473-484 (2016).

29. Ahmad, S. et al. Disentangling the biological pathways involved in early features of Alzheimer's disease in the Rotterdam Study. Alzheimers Dement. 14 848-857 (2018)

30. Dudbridge, F. Power and predictive accuracy of polygenic risk scores. PLoS Genet. 9, e1003348 (2013).

31. Freudenberg-Hua, Y., Li, W. \& Davies, P. The role of genetics in advancing precision medicine for Alzheimer's disease-a narrative review. Front. Med. 5 108 (2018).

32. Huisman, M. et al. Cohort profile: the longitudinal aging study Amsterdam. Int. J. Epidemiol. 40, 868-876 (2011).

33. Hoogendijk, E. O. et al. The longitudinal aging study Amsterdam: cohort update 2016 and major findings. Eur. J. Epidemiol. 31, 927-945 (2016).

34. van der Flier, W. M. \& Scheltens, P. Amsterdam dementia cohort: performing research to optimize care. J. Alzheimers Dis. 62, 1091-1111 (2018).

35. van der Flier, W. M. et al. Optimizing patient care and research: the Amsterdam Dementia Cohort. J. Alzheimers Dis. 41, 313-327 (2014).

36. Rademaker, M. C., de Lange, G. M., Palmen, S. J. M. C. The Netherlands Brain Bank for Psychiatry. Handb. Clin. Neurol. 150, 3-16 (2018). 
37. Lambert, J.-C. et al. Genome-wide association study identifies variants at CLU and CR1 associated with Alzheimer's disease. Nat. Genet. 41, 1094-1099 (2009).

38. Strittmatter, W. J. et al. Apolipoprotein E: high-avidity binding to beta-amyloid and increased frequency of type 4 allele in late-onset familial Alzheimer disease. Proc. Natl Acad. Sci. USA 90, 1977-1981 (1993).

39. Corder, E. H. et al. Protective effect of apolipoprotein E type 2 allele for late onset Alzheimer disease. Nat. Genet. 7, 180-184 (1994).

40. Jansen I. E., et al. Genome-wide meta-analysis identifies new loci and functional pathways influencing Alzheimer's disease risk. Nat Genet. 51, 404-413 (2019).

41. Das, S. et al. Next-generation genotype imputation service and methods. Nat. Genet. 48, 1284-1287 (2016).

42. McCarthy, S. et al. A reference panel of 64,976 haplotypes for genotype imputation. Nat. Genet. 48, 1279-1283 (2016).

43. 1000 Genomes Project Consortium. et al. A global reference for human genetic variation. Nature 526, 68-74 (2015).

44. Jefferies, W. A. et al. Adjusting the compass: new insights into the role of angiogenesis in Alzheimer's disease. Alzheimers Res. Ther. 5, 64 (2013).

45. Vagnucci, A. H. \& Li, W. W. Alzheimer's disease and angiogenesis. Lancet 361, 605-608 (2003).

46. Watanabe K, Taskesen E, van Bochoven A, Posthuma D. Functional mapping and annotation of genetic associations with FUMA. Nat Commun. 8, 1826 (2017).

47. Ashburner, M. et al. Gene ontology: tool for the unification of biology. The Gene Ontology Consortium. Nat. Genet. 25, 25-29 (2000).

48. The Gene Ontology Consortium. Expansion of the Gene Ontology knowledgebase and resources. Nucleic Acids Res. 45, D331-D338 (2017).

49. Huang, D. W., Sherman, B. T. \& Lempicki, R. A. Systematic and integrative analysis of large gene lists using DAVID bioinformatics resources. Nat. Protoc. 4 , 44-57 (2009).

50. Huang, D. W., Sherman, B. T. \& Lempicki, R. A. Bioinformatics enrichment tools: paths toward the comprehensive functional analysis of large gene lists. Nucleic Acids Res. 37, 1-13 (2009).

51. Escott-Price, V., Myers, A. J., Huentelman, M. \& Hardy, J. Polygenic risk score analysis of pathologically confirmed Alzheimer disease: PRS analysis of AD. Ann. Neurol. 82, 311-314 (2017).

52. Escott-Price, V., Shoai, M., Pither, R., Williams, J. \& Hardy, J. Polygenic score prediction captures nearly all common genetic risk for Alzheimer's disease. Neurobiol. Aging 49, 214.e7-214.e11 (2017).
53. DESGESCO (Dementia Genetics Spanish Consortium), EADB (Alzheimer Disease European DNA biobank) et al. A nonsynonymous mutation in PLCG2 reduces the risk of Alzheimer's disease, dementia with Lewy bodies and frontotemporal dementia, and increases the likelihood of longevity. Acta Neuropathol. (Berl). https://doi.org/10.1007/s00401-01902026-8 (2019)

54. For the International Genomics of Alzheimer's Project et al. Evaluation of a genetic risk score to improve risk prediction for Alzheimer's disease. J. Alzheimers Dis. 53, 921-932 (2016).

55. Chaudhury $\mathrm{S}$, et al. Alzheimer's disease polygenic risk score as a predictor of conversion from mild-cognitive impairment. Transl Psychiatry. 9, 154 (2019).

56. van der Lee, S. J. et al. The effect of APOE and other common genetic variants on the onset of Alzheimer's disease and dementia: a community-based cohort study. Lancet Neurol. 17, 434-444 (2018).

57. Leonenko, G. et al. Genetic risk for Alzheimer's disease is distinct from genetic risk for amyloid deposition. Ann. Neurol. https://doi.org/10.1002/ana.25530 (2019)

58. Ransohoff, R. M. How neuroinflammation contributes to neurodegeneration. Science 353, 777-783 (2016).

59. Heneka, M. T. et al. Neuroinflammation in Alzheimer's disease. Lancet Neurol. 14, 388-405 (2015).

60. Joshi, P. K. et al. Genome-wide meta-analysis associates HLA-DQA1/ DRB1 and LPA and lifestyle factors with human longevity. Nat Commun. 8, 910 (2017).

61. Srinivasan, K. et al. Untangling the brain's neuroinflammatory and neurodegenerative transcriptional responses. Nat Commun. 7, 11295 (2016).

62. Wang, Y. et al. TREM2-mediated early microglial response limits diffusion and toxicity of amyloid plaques. J. Exp. Med. 213, 667-675 (2016).

63. Orre, M. et al. Isolation of glia from Alzheimer's mice reveals inflammation and dysfunction. Neurobiol. Aging 35, 2746-2760 (2014).

64. Hansen, D. V., Hanson, J. E. \& Sheng, M. Microglia in Alzheimer's disease. J. Cell Biol. 217, 459-472 (2018).

65. Timmers, P. R. et al. Genomics of 1 million parent lifespans implicates nove pathways and common diseases and distinguishes survival chances. Elife. 8, e39856 (2019).

66. Davies, G. et al. Study of 300,486 individuals identifies 148 independent genetic loci influencing general cognitive function. Nat Commun. 9, 2098 (2018). 